\title{
Immune status could be associated with severity in COVID-19 patients
}

\author{
(iD) Chuan Liu', \\ (iD) iahui Yang ${ }^{3}$ \\ D. Wancong Wang ${ }^{1}$ \\ (iD) Yang Mi,2 \\ (D) Youcai Tang ${ }^{2,4}$ \\ (iD) Pengyuan Zheng ${ }^{1,2}$
}

\begin{abstract}
1.Department of Gastroenterology, The Fifth Affiliated Hospital of Zhengzhou University, Zhengzhou, 450052, Henan Province, China. 2.Marshall B.J.Medical Research Center of Zhengzhou University, Zhengzhou, 450052, Henan Province, China. 3. Department of Geriatric, West China Hospital of Sichuan University, Chengdu, 610041, Sichuan Province, China. 4.Department of Children's Rehabilitation Medicine, The Fifth Affliated Hospital of Zhengzhou University, Zhengzhou, 450052, Henan Province, China.
\end{abstract}

KEYWORDS: Coronavirus Infections/immunology. Meta-analysis. Immunocompetence. Immunologic Factors.

Dear Editor,

Corona Virus Disease 2019 (COVID-19) is a highly contagious disease that has caused outbreaks across the globe. As of May 21, 2020, a total of 5,027,732 confirmed COVID-19 cases have been reported in 188 countries, with an average mortality of $6.54 \%^{1}$. The current relationship between COVID-19 disease severity and immune function status is unclear. As far as we know, this study is the first meta-analysis to compare immune status and immunoglobulin efficacy in severe vs. non-severe patients with COVID-19.

An extensive electronic search of PubMed, Embase, and Cochrane Library was conducted without date limitations (until May 6, 2020), using the keywords "COVID-19”, “2019-nCoV”, "SARS-CoV-2”,
“Novel Coronavirus", "Feature”, "Immune”, etc. Two authors independently screened articles, assessed qualities, and performed data extraction. Any discrepancies during these steps were regularly resolved by consulting with a third reviewer. Severe COVID-19 infection was defined as severe pneumonia, acute respiratory distress syndrome (ARDS), treatment with mechanical ventilation or intensive care unit (ICU), and/or death. Dichotomous variables were estimated with Odd Risks (OR) and 95\% confidence interval (CI). Continuous data (using mean and standard deviation - SD) were compared and the mean difference (MD) was calculated with 95\% $\mathrm{CI}$, and a P-value $<0.05$ was considered statistically 


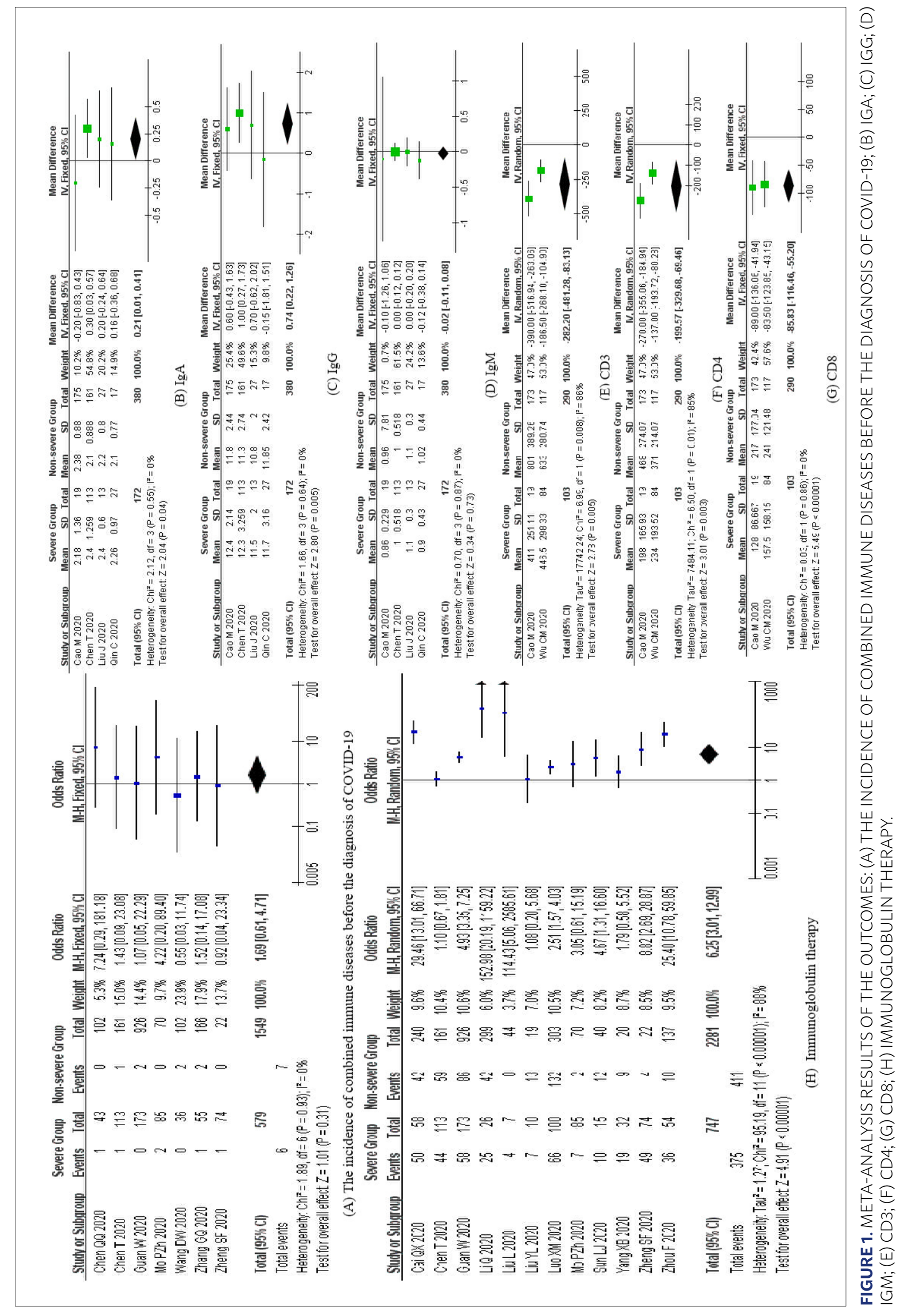


significant. The random-effects model was used if $\mathrm{I}^{2}>50 \%$. The publication bias was evaluated by visual inspection of the funnel plot. Our meta-analysis was carried out using Review Manager software version 5.3 (Cochrane Collaboration).

Nineteen studies ${ }^{2-20}$ were enrolled in our analysis. All studies originated from China and involved 4,011 confirmed COVID-19 patients in total. The incidence of combined immune diseases before the diagnosis of COVID-19 was not significantly associated with an enhanced risk of severe COVID-19 (OR: 1.69, 95\% CI: 0.61-4.71, P=0.31). Immune globulin A (IgA) and immune globulin $\mathrm{G}(\mathrm{IgG})$ were found to be increased in COVID-19 patients in the severe group [IgA: $\mathrm{MD}=0.21$, 95\% CI (0.01, 0.41), $\mathrm{P}=0.04$; IgG: $\mathrm{MD}=0.74,95 \% \mathrm{CI}(0.22$, 1.26), $\mathrm{P}=0.005$ ]. Immune globulin $\mathrm{M}$ (IgM) was not found to be correlated with the severity of COVID-19 (MD: -0.02, 95\% CI: -0.11, 0.08, P=0.73). Our study revealed that a decrease in the mean cluster of differentiation (CD)3, CD4 and CD8 was significantly associated with an increased likelihood of severity [CD3: $\mathrm{MD}=-282.2$, 95\%CI (-481.28, -83.13), $\mathrm{P}=0.005$; CD4: MD=-199.57, 95\%CI (-329.68, -69.46), $\mathrm{P}=0.003$; CD8: $\mathrm{MD}=-85.83,95 \% \mathrm{CI}(-116.46,-55.20), \mathrm{P}<0.0001]$. Immunoglobulin therapy is significantly more effective in severe COVID-19 patients (OR: 6.25, 95\% CI: 3.01-12.99, $\mathrm{P}<0.0001)$. Based on a visual inspection of the funnel plots, no evidence of publication bias was found. More details of our meta-analysis are presented in Figure 1.

This meta-analysis provides evidence that hypoimmune states could be associated with a more severe form of COVID-19, and that immunoglobulins are more effective against severe COVID-19. Our results are consistent with some previous descriptive reviews ${ }^{21-23}$. In addition, this study has important clinical implications. First, it can potentially help in disease assessment as the COVID-19 pandemic continues to affect multiple countries. Second, it reminds us of the relevance of early initiation of immunoglobulin therapy, which is effective in improving the prognosis of severe and critical type patients.

This study has its limitations. First, most of the studies included are retrospective and lack a prospective design. Second, some of the outcomes in this study had a high I2 value, which represents heterogeneity. This might be explained by the small sample size, limited data, and different patient populations.

Based on our results, a hypoimmune status might be an important sign of COVID-19 severity, guiding clinicians to identify severe patients earlier and faster and alerting clinicians that immunoglobulins have better efficacy in critically ill patients. Further, more high-quality literature is needed to better support this conclusion.

\section{Acknowledgments}

The National Natural Science Foundation of China (No. 81370494 and No. 31471330) funded this manuscript.

\section{Ethical approval}

All analyses were based on previously published studies, thus no ethical approval and patient consent are required.

\section{Author's Contribution}

Chuan Liu conceptualized and designed the study, drafted the initial manuscript, and approved the final manuscript as submitted. Jiahui Yang, Wancong Wang, and Yang Mi obtained the data, carried out the analyses. Youcai Tang and Pengyuan Zheng critically reviewed the manuscript and approved the final manuscript as submitted.

\section{Conflict of Interest}

The authors declare no conflict of interest.

\section{REFERENCES}

1. Johns Hopkins University. COVID-19 Dashboard by the Center for Systems Science and Engineering (CSSE) at Johns Hopkins University (JHU). Baltimore: Johns Hopkins University; 2020. [cited 2020 May 21]. Available from: https://gisanddata.maps.arcgis.com/apps/opsdashboard/index.html\#/ bda7594740fd40299423467b48e9ecf6
2. Cai Q, Huang D, Ou P, Yu H, Zhu Z, Xia Z, et al. COVID-19 in a designated infectious diseases hospital outside Hubei Province, China. Allergy. 2020;75(7):1742-52.

3. Chen Q, Zheng Z, Zhang C, Zhang X, Wu H, Wang J, et al. Clinical characteristics of 145 patients with corona virus disease 2019 (COVID-19) in 
Taizhou, Zhejiang, China. Infection. 2020;1-9.

4. Chen T, Wu D, Chen H, Yan W, Yang D, Chen G, et al. Clinical characteristics of 113 deceased patients with coronavirus disease 2019: retrospective study. Bmj. 2020;368:m1091.

5. Guan WJ, Ni ZY, Hu Y, Liang WH, Ou CQ, He JX, et al. Clinical Characteristics of Coronavirus Disease 2019 in China. The New England journal of medicine. 2020;382(18):1708-20.

6. Li Q, Zhang J, Ling Y, Li W, Zhang X, Lu H, et al. A simple algorithm helps early identification of SARS-CoV-2 infection patients with severe progression tendency. Infection. 2020;1-8.

7. Liu J, Li S, Liu J, Liang B, Wang X, Wang H, et al. Longitudinal characteristics of lymphocyte responses and cytokine profiles in the peripheral blood of SARS-CoV-2 infected patients. EBioMedicine. 2020;55:102763.

8. liu lei GJ-y. Clinical characteristics of 51 patients discharged from hospital with COVID-19 in Chongqing-China. medrxiv. 2020.

9. Luo X, Zhou W, Yan X, Guo T, Wang B, Xia H, et al. Prognostic value of C-reactive protein in patients with COVID-19. Clinical infectious diseases: an official publication of the Infectious Diseases Society of America. 2020; ciaa641.

10. Mo $P$, Xing Y, Xiao Y, Deng L, Zhao Q, Wang H, et al. Clinical characteristics of refractory COVID-19 pneumonia in Wuhan, China. Clinical infectious diseases: an official publication of the Infectious Diseases Society of America. 2020; ciaa270.

11. Qin C, Zhou L, Hu Z, Zhang S, Yang S, Tao Y, et al. Dysregulation of immune response in patients with COVID-19 in Wuhan, China. Clinical infectious diseases: an official publication of the Infectious Diseases Society of America. 2020;71(15):762-68.

12. Sun L, Shen L, Fan J, Gu F, Hu M, An Y, et al. Clinical Features of Patients with Coronavirus Disease 2019 (COVID-19) from a Designated Hospital in Beijing, China. Journal of medical virology. 2020;10.1002/jmv.25966.

13. Wang D, Hu B, Hu C, Zhu F, Liu X, Zhang J, et al. Clinical Characteristics of 138 Hospitalized Patients With 2019 Novel Coronavirus-Infected Pneumonia in Wuhan, China. Jama. 2020;323(11):1061-69.
14. Wu $C$, Chen $X$, Cai $Y, X i a \mid$, Zhou $X, X u S$, et al. Risk Factors Associated With Acute Respiratory Distress Syndrome and Death in Patients With Coronavirus Disease 2019 Pneumonia in Wuhan, China. JAMA internal medicine. 2020;180(7):1-11.

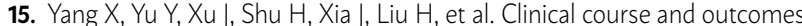
of critically ill patients with SARS-CoV-2 pneumonia in Wuhan, China: a single-centered, retrospective, observational study. The Lancet Respiratory medicine. 2020;8(5):475-81.

16. Zhang G, Hu C, Luo L, Fang F, Chen Y, Li I, et al. Clinical features and shortterm outcomes of 221 patients with COVID-19 in Wuhan, China. Journal of clinical virology: the official publication of the Pan American Society for Clinical Virology. 2020;127:104364.

17. Zheng S, Fan I, Yu F, Feng B, Lou B, Zou Q, et al. Viral load dynamics and disease severity in patients infected with SARS-CoV-2 in Zhejiang province, China, January-March 2020: retrospective cohort study. Bmj. 2020;369:m1443

18. Zhou F, Yu T, Du R, Fan G, Liu Y, Liu Z, et al. Clinical course and risk factors for mortality of adult inpatients with COVID-19 in Wuhan, China: a retrospective cohort study. Lancet. 2020;395(10229):1054-62.

19. Cao M, Zhang D, Wang Y, Lu Y, Zhu X, Li Y, et al. Clinical Features of Patients Infected with the 2019 Novel Coronavirus (COVID-19) in Shanghai, China. medRxiv. 2020

20. Liu $Y$, Sun W, Li I, Chen L, Wang Y, Zhang L, et al. Clinical features and progression of acute respiratory distress syndrome in coronavirus disease 2019. medRxiv. 2020

21. Lin L, Lu L, Cao W, Li T. Hypothesis for potential pathogenesis of SARSCoV-2 infection: a review of immune changes in patients with viral pneumonia. Emerg Microbes Infect. 2020;9(1):727-32.

22. Kadkhoda K. COVID-19: an immunopathological view. mSphere 2020;5(2):e00344-20.

23. Jawhara S. Could intravenous immunoglobulin collected from recovered coronavirus patients protect against COVID-19 and strengthen the immune system of new patients? Int | Mol Sci. 2020;21(7):2272. 